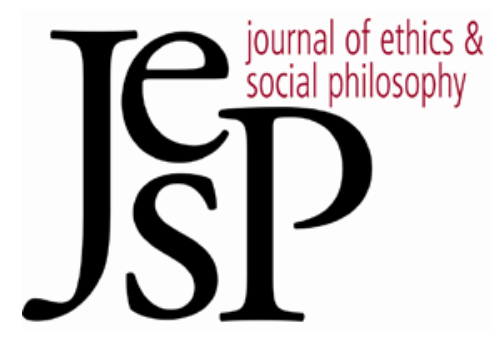

DISCUSSION NOTE

\title{
Money Pumps, Diachronic and Synchronic
}

\author{
BY YAIR LEVY
}

JOURNAL OF ETHICS \&SOCIAL PHILOSOPHY

Discussion Note | OCTOBER 2014

URL: WWW.JESP.ORG

COPYRIGHT (C) YAIR LEVY 2014 


\title{
Money Pumps, Diachronic and Synchronic
}

\author{
Yair Levy
}

W HY HAVE ACYCLIC PREFERENCES? A common reply invokes the threat of being turned into a money pump. If one prefers $a$ over $b, b$ over $c$, but $c$ over $a$, one may be manipulated into paying for a series of trades, only to be left with one's initial object of choice. This influential argument for acyclicity of preferences appeared first in Davidson, McKinsey and Suppes (1955). In their example, Mr. S. has the following cyclic set of preferences: Faced with choices among the positions of full professor with a salary of $\$ 5,000$ (a); associate professor at $\$ 5,500$ (b); and assistant professor at $\$ 6,000$ (c), Mr. S. prefers $a$ over $b$, $b$ over $c$ and $c$ over $a$. The cyclic set renders Mr. S. vulnerable to manipulation:

The department head, advised of Mr. S’s preferences, says, “I see you prefer $b$ to $c$, so I will let you have the associate professorship - for a small consideration. The difference must be worth something to you." Mr. S. agrees to slip the department head $\$ 25$ to get the preferred alternative. Now the department head says, "Since you prefer $a$ to $b$, I'm prepared - if you will pay me a little for my trouble - to let you have the full professorship." Mr. S. hands over another $\$ 25$ and starts to walk away, well satisfied, we may suppose. "Hold on," says the department head, "I just realized you'd rather have $c$ than $a$. And I can arrange that - provided ..."1

A common reaction to the money-pump argument raises the possibility of devising ways to circumvent the threat of exploitation. For example, having traded $c$ for $b$ and then $b$ for $a$, Mr. S. may "get the drift" and refuse any further transactions with the greedy department head (Schick 1986); alternatively, he may resolve in advance to avoid any trades that will result in his being exploited (McClennen 1990: 13).

Recently, a new version of Money Pump has surfaced, which promises to improve on the original when it comes to overcoming these standard objections. Thus Johan Gustafsson (2013) dismisses the above objections as "irrelevant"; their seeming force comes from targeting a conspicuous but dispensable feature of the argument, while leaving its important core untouched. The threat of being fleeced for money is a mere dramatic device that serves to illustrate the deeper lesson of Money Pump: the irrationality of cyclic preferences. Remove the money pumper from the scene and you remove a vivid, dramatic illustration of why cyclic preferences are rationally flawed; but you have not upset the thrust of the argument. Gustafsson demonstrates this point by formulating a synchronic analogue of the diachronic money pump. His synchronic version dispenses with the threat of exploitation, and hence undercuts the standard objections. Instead of the sequence of pairwise choices that figures in the original diachronic argument, Mr. S. is imagined to face just a single choice from the complete three-term set $\{a, b, c\}$. With no trades offered to swap one object for another, Mr. S. is not vulnerable to manipulation by the department head, and consequently has no use for precautionary measures of the kind suggested by Schick and McClennen. But his irrationality re-

1 Ibid.: 146. 
mains manifest. His cyclic preferences render him unable to make a choice with which he will be satisfied; whatever he chooses, there will be some alternative that he prefers.

The synchronic version of Money Pump demonstrates the dialectical dispensability of the threat of exploitation. The irrationality of Mr. S. consists in a defective structure of preferences that cannot perform its role of effecting a stable choice. It is because of this irrational defect that Mr. S. is vulnerable to exploitation in the original money pump: No matter which option he manages to secure, he would always be willing to pay to have another one instead. His vulnerability is thus indicative, not constitutive, of the irrational defect: Even in the absence of the opportunity to pay for trades, the impossibility of making a satisfactory choice from the threeterm set exposes the real problem with cyclic preferences.

It is certainly correct that vulnerability to manipulation does not exhaust the irrationality that Money Pump is thought to demonstrate. A similar point has been noted in the literature on another prominent argument for rational coherence that likewise invokes the threat of exploitation - the Dutch-book argument for having credences that satisfy the axioms of probability, for fear that otherwise one would be disposed to accept a losing profile of bets. Here is David Lewis (1999: 404-5):

\begin{abstract}
Note also that the point of any Dutch book argument is not that it would be imprudent to run the risk that some sneaky Dutchman will come and drain your pockets. After all, there aren't so many sneaky Dutchmen around; and anyway, if ever you see one coming, you can refuse to do business with him. Rather, the point is that if you are vulnerable to a Dutch book, whether synchronic or diachronic, that means that you have two contradictory opinions about the expected value of the very same transaction. To hold contradictory opinions may or may not be risky, but it is in any case irrational.
\end{abstract}

Lewis is concerned with Dutch books, but parallel observations can be made about money pumps. The passage records two possible responses to the argument: that it trades on circumstances (the presence of Dutch bookies/money pumpers) that are too exotic to ground a perfectly general reason for avoiding irrationality; and that, even when such circumstances are in place, the threat they pose is easily neutralized without resolving the associated irrationality - a point demonstrated by Schick and McClennen for Money Pump when they propose ways to circumvent exploitation. Now the synchronic version of Money Pump successfully defuses both kinds of objection as formulated to target the diachronic version of the argument; with this it may seem to reveal the underlying irrational flaw of cyclic preferences, similarly to how Lewis points to the deeper flaw underlying the vulnerability to Dutch books. However, it is possible to recover isomorphic objections that target the synchronic money pump itself.

To bring this possibility into view, notice first a seemingly innocuous yet crucial assumption made by the synchronic money pump. The imputation of inability to effect a stable choice only gains purchase if we assume that one's pairwise preferences imply a corresponding pattern of preference over the three-term set $\{a, b, c\}$; otherwise, no grounds exist for ascribing any specific three-term preference pattern, and in particular not the allegedly unstable one. What warrants the assumption? Gustafsson 
himself comes close to noticing this gap in his argument when he discusses Arrow's restriction of preference to pairwise choices (Arrow 1959). But his reply mishandles the challenge:

\footnotetext{
This objection, however, assumes that we must know what would happen if Mr. S were faced with a choice from $\{a, b, c\}$. But we only need to know that he would choose something, and that part is easy - we can just stipulate that the situation is such that the agent is forced to choose an alternative. The point is that we do not need to know which of $a, b$, and $c \mathrm{Mr}$. S would choose, since he would [choose an alternative to which another is preferred] whichever he chooses.
}

The problem is not so easily removed, however. Stipulating that one must make a choice from the three-term set may be enough to ensure that one will choose one of the options; but it says nothing about what one prefers. Gustafsson suggests that we need not know which specific choice one will actually make, since for any such choice some other one will be considered preferable. With this he has gone beyond a mere stipulation that some choice or other will be made; he is also claiming that any such choice is guaranteed to be suboptimal. And the measure of suboptimality is precisely one's pairwise preferences. If Mr. S's three-term preference were not determined by his pairwise preferences, he would not be condemned to choosing a suboptimal alternative.

The point bears notice, as it clears ground for reconstructing versions of the original objections to Money Pump. Recall the two objections encapsulated in the passage from Lewis. They are: (a) that the presence of money pumpers is too remote a contingency to ground a general reason for acyclic preferences; and (b) that the contingency, even when it does occur, carries a threat that is easily defused without resolving the associated irrationality. Start with (b). The original version of the objection, as formulated by McClennen, Schick and others, suggests that exercising minimal forethought can eliminate the threat of exploitation; if $\mathrm{Mr}$. S. refused to do business with the department head or planned his transactions in advance, he could not be fleeced for money. Now the threat posed by the synchronic money pump is that of facing a choice from the three-term set $\{a, b, c\}$ that is guaranteed to be suboptimal. And this threat is likewise circumvented by some forward thinking. Mr. S. may execute a protocol for choosing from $\{a, b, c\}$ that does not involve simply reading his choice off his pairwise preferences. For example, he may decide to settle on some option randomly, or he may take account of the different (wider) context of choice presented by $\{a, b, c\}$.

If the procedure seems contrived, notice that many choice problems are in fact such that preference varies with context. And, in those cases, one may have cyclic preferences and yet be perfectly able to execute a satisfactory choice from the three-term set. Take a case described by John Broome (1991, \5.4; 1999). Given a choice between mountaineering in the Alps $(M)$ and visiting Rome $(R)$, Maurice prefers to visit Rome. Given a choice between visiting Rome and staying at home $(H)$, Maurice prefers to stay at home. Finally, given a choice between staying home and going mountaineering, he prefers mountaineering. As stated, Maurice's preferences are cyclic; he prefers $\mathrm{R}$ over $M, M$ over $H$, but $H$ over $\mathrm{R}$. His ra- 
tionale for these preferences is that staying at home when the alternative is going mountaineering will seem cowardly, and so he prefers mountaineering. But when the alternative to mountaineering is visiting Rome, choosing Rome would seem cultured rather than cowardly, so he prefers Rome (Broome 1991, \5.4; 1999).

Faced with a choice among $\{M, R, H\}$, Maurice does not suffer the inescapable dissatisfaction imputed to him by the synchronic money pump. For example, he may happily choose $R$, reasoning that visiting Rome would not seem cowardly but cultured. Scenarios like Maurice's, where choice varies with context, are ubiquitous. And this reconstructs an isomorphic objection to (a) above: Just like its diachronic counterpart, the synchronic money pump also trades on rare circumstances.

The above point may be put differently by noting that the synchronic money pump relies on the dubious Weak Axiom of Revealed Preference (WARP), sometimes invoked by decision theorists, whereby if any choice situation exists in which one chooses $a$ rather than $b$, then in no choice situation may one choose $b$ when $a$ is also available. ${ }^{2}$ Of course, if the preference relation is understood as consisting in (or at least entailing) WARP, then the synchronic money pump goes through: As stated, Maurice's preferences obviously violate WARP. But the present point is precisely that the possibility of agents like Maurice brings out the implausibility of understanding preference in that way. ${ }^{3}$

The reconstruction of the original objections to Money Pump may seem to fail, as context cuts in already at the pairwise stage, rendering the charge of cyclic preferences spurious. Writing $H_{r}$ for "staying at home when the alternative is going to Rome," and $H_{m}$ for "staying at home when the alternative is going mountaineering," Maurice's pairwise preferences should be understood as $\mathrm{R}$ over $M, M$ over $H_{m}$ and $H_{r}$ over R. Individuated finely in this way, no alternative involving staying at home appears twice, and Maurice cannot be understood as having cyclic preferences. He is thus no longer a target of Money Pump. ${ }^{4}$

However, as Broome points out (1991: 100-7; 1999: 73-75), the strategy of contextually refining the alternatives cannot plausibly be applied across the board. If every case of apparent cyclicity can be explained away by refining the individuation of alternatives, acyclicity becomes a constraint that cannot be violated, hence empty. There must therefore be cases in which the identity of the unchosen alternative cannot rationally factor into one's preference. For some $A, B$ and $C$, that is, it must be the case that, unlike Maurice, one treats $C_{a}$ and $C_{b}$ as equivalent, if acyclicity is to have any bite..$^{5}$

\footnotetext{
2 See for example Sen $(1971 ; 1993)$. In fact, the strictly weaker assumption, known variously in the literature as Sen's Property $\alpha$, Chernoff's Condition, and Independence of Irrelevant Alternatives, would seem to suffice for the purposes of the synchronic money pump.

${ }^{3}$ I am grateful to an anonymous referee for getting me to address this issue.

${ }^{4}$ For a related discussion of preference redescription in the context of Sen's $\alpha$, see Neumann (2007).

${ }^{5}$ As Broome notes, a different possible solution to the problem of emptiness would be to require rational indifference between $C_{a}$ and $C_{b}$. I shall set aside this alternative solution
} 
Take an exemplar of this sort, then. One prefers $A$ over $B, B$ over $C_{b}$ and $C_{a}$ over $A$. Suppose the only difference one can detect between $C_{a}$ and $C_{b}$ is the following "Cambridge difference": To choose the former is to choose $C$-rather-than- $A$, whereas to choose the latter is to choose $C$ rather-than- $B$. One is then presumably required to treat both alternatives as equivalent. Given that one prefers $C_{a}$ over $A$, and $A$ over $B$, acyclicity requires that one prefer $C_{a}$ over $B$. Acyclicity also requires, given that one treats $C_{a}$ and $C_{b}$ as equivalent, that one prefer $C_{b}$ over $B$. But one actually prefers $B$ over $C_{b}$. One is hence violating acyclicity, and is a target of Money Pump, unlike perhaps Maurice.

Now does one face inescapable dissatisfaction given a choice from $\{A, B, C\}$, as the synchronic money pump predicts? There are no grounds for supposing that one does. The way context informs one's pairwise preferences need not constrain one's three-term preference. Thus, while one is required to treat $C_{a}$ as equivalent to $C_{b}$, one may regard $C_{a, b}$ differently. And so one can happily choose $A$, for example, reasoning that although one prefers $C_{a}$ to $A$, one does not have a preference for $C_{a, b}$ over $A$ and $B$.

With this, parallel objections to the original money pump have been recovered for the synchronic version. One's pattern of pairwise preferences often fails to imply a corresponding three-term preference - for example, when context influences choice; hence the threat that cyclic preferences condemn one to suboptimal choice arises only rarely. And even when the threat does arise, it can be defused by taking care to choose in some way other than being guided by one's pairwise preferences, while remaining irrational. The synchronic money pump strips away the dramatic device of exploitation to reveal the core defect allegedly demonstrated by the original diachronic version; but the standard objections to the latter can likewise be stripped of the drama to target that core directly.

If the parallel between the reformulated objections and those made originally by Schick and McClennen seems loose, notice that the main criticism of the synchronic money pump is unaffected. The possibility of executing a satisfactory choice from a three-term set while remaining in violation of acyclicity draws out the failure of synchronic money pump to demonstrate any rational defect with cyclic preferences. The synchronic version of the argument thus does not improve on the original: A failure of extensional adequacy vitiates money pump, in both its diachronic and synchronic forms. ${ }^{\circ}$

Yair Levy

University of Oxford

Department of Philosophy

yairik@gmail.com

here, as Broome himself does, so as to avoid complication and retain continuity with others who have discussed the problem (e.g., Tversky 1975: 170-73).

${ }^{6}$ For very helpful discussion and comments on previous drafts, I am grateful to John Broome, Johan Gustafsson, Uri Leibowitz, Arnon Levy, Ittay Nissan and an anonymous referee. 


\section{References}

Arrow, K. J. (1959) "Rational Choice Functions and Orderings," Economica 26: 121-27.

Broome, J. (1999) "Can a Humean Be Moderate?" in Ethics Out of Economics, Oxford: Oxford University Press, pp. 68-87.

(1991) Weighing Goods, Oxford: Basil Blackwell.

Davidson, D., et al. (1955) "Outlines of a Formal Theory of Value, I," Philosophy of Science 22: $140-60$.

Gustafsson, J. E. (2013) “The Irrelevance of the Diachronic Money-Pump Argument for Acyclicity," Journal of Philosopby 110: 460-64.

Lewis, D. (1999) "Why Conditionalize?" in Papers in Metaphysics and Epistemology, Vol. II, Cambridge: Cambridge University Press.

McClennen, E. F. (1990) Rationality and Dynamic Choice: Foundational Explorations, Cambridge: Cambridge University Press.

Neumann, M. (2007) "Choosing and Describing: Sen and the Irrelevance of Independence Alternatives," Theory and Decision 63: 79-94.

Schick, F. (1986) "Dutch Bookies and Money Pumps," Journal of Philosophy 83: 112-19.

Sen, A. (1993) "Internal Consistency of Choice," Econometrica 61: 495-521. (1971) "Choice Functions and Revealed Preference," The Review of Economic Studies 38: 307-17.

Tversky, A. (1975) "A Critique of Expected Utility Theory: Descriptive and Normative Considerations," Erkenntnis 9: 163-73. 